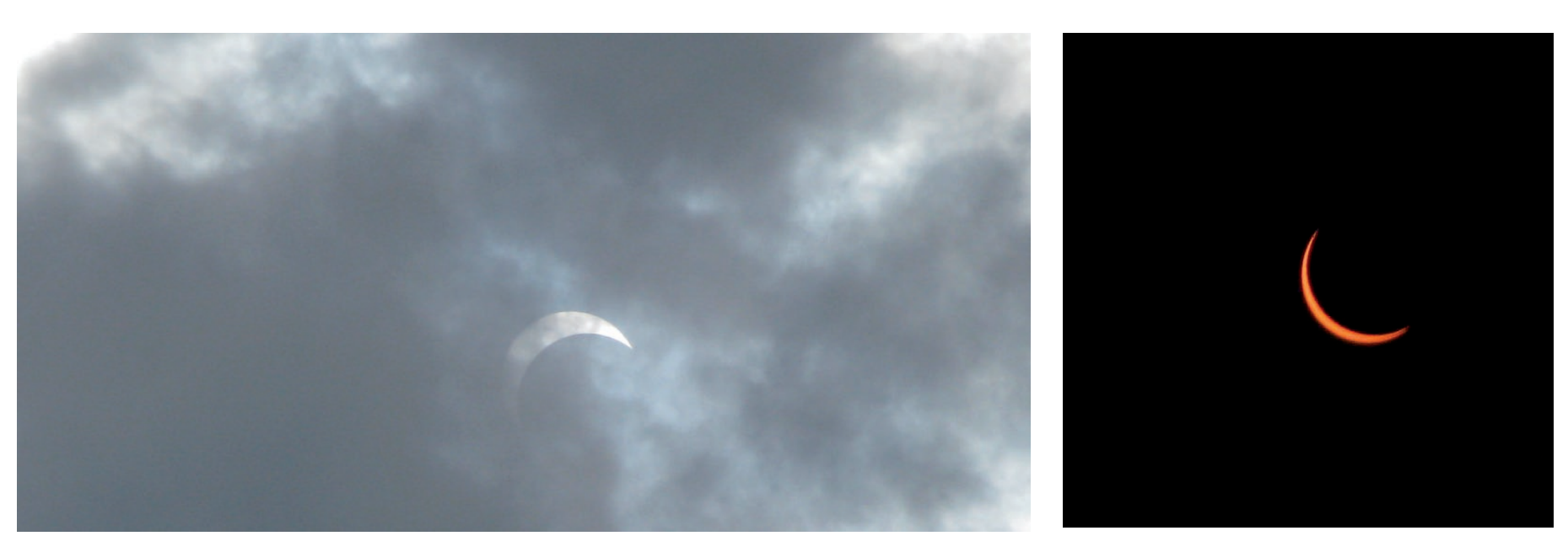

L'éclipse de Soleil à Franceville, le 3 novembre 2013, à travers les nuages à 14 h 42 (à gauche), puis à travers les lunettes à 15 h 01 (à droite).

\title{
Éclipse solaire au Gabon en novembre 2013
}

À Franceville (Masuku) au Gabon, une semaine d'animations menée par les universités d'Aix-Marseille et de Masuku a débouché sur une grande fête de la physique et une observation collective de l'éclipse solaire le 3 novembre 2013. Plus de 200 élèves ont participé aux ateliers, et plus de 2000 personnes se sont arrêtées devant le podium installé en centre-ville pour l'occasion et ont admiré la Lune " grignotant» le Soleil.

Ce projet a été soutenu financièrement par la Société Française de Physique.

Le spectaculaire événement qu'est une éclipse solaire est toujours l'occasion d'expliquer au grand public les phénomènes d'ombre et de discuter du système solaire. En faire une action en Afrique garantit d'attirer des foules et promet une belle fête.

Dans le cadre d'une collaboration existante entre les universités d'Aix-Marseille (AMU) et de Masuku (USTM, l'université de sciences du Gabon, voir l'encadré), à Franceville dans la province du Haut-Ogooué et en collaboration avec l'écomusée de Franceville, une grande semaine d'animation a fait le bonheur des petits et des grands.

Étudiants, élèves et habitants ont pu assister à la semaine de l'éclipse, du 28 octobre au 3 novembre 2013. Durant cette semaine, toute une série d'animations a été organisée, débouchant sur une grande fête de la physique et une observation collective de l'éclipse de Soleil le 3 novembre.

Quatre ateliers, organisés sur le site de l'université, ont permis aux élèves de collèges et d'écoles primaires de découvrir par eux-mêmes certains phénomènes physiques avec l'aide d'enseignants d'AMU et de l'USTM. Les étudiants du tout récent Master de Physique de l'université de Masuku ont été très impliqués, tant sur les aspects scientifiques que pour la logistique.

Les élèves des écoles primaires ont fabriqué des cadrans solaires et modélisé le phénomène de l'éclipse autour d'une mappemonde. Pour l'atelier "cadran solaire ", les élèves ont montré toutes leurs qualités artistiques en décorant magnifiquement leurs cadrans (fig. 1). Des livres d'astronomie pour enfants étaient à leur disposition et c'était l'occasion de nombreuses questions. Pour l'atelier « autour d'une mappemonde ", un vidéoprojecteur représentant le Soleil, une balle représentant la Lune, et la mappemonde ont permis d'expliquer l'alternance jour-nuit, les phases de la Lune, les éclipses de Soleil et de Lune. Plusieurs phénomènes tels que la forme de la Terre et les fuseaux horaires ont été compris par les enfants à l'aide de ce dispositif. Par contre, la notion de variation de la durée du jour et de la nuit selon les saisons est plus compliquée à discuter. En effet, au Gabon, le Soleil se lève invariablement à $6 \mathrm{~h}$ et " tombe » derrière l'horizon à $18 \mathrm{~h}$. D'abord spectateurs, les étudiants de l'université se sont approprié les ateliers.

Les deux ateliers mis en place pour les élèves des collèges avaient pour thèmes la spectroscopie et la fabrication de maquettes permettant de modéliser l'éclipse. L'atelier «spectroscopie " s'est organisé autour de l'observation de spectres de différentes sources. L'atelier "fabrication de maquette " a consisté à faire construire aux adolescents le système Terre-Lune en maquette à l'aide de boules en polystyrène de plusieurs tailles, pour ensuite observer une éclipse sur ces maquettes à l'aide du Soleil (fig. 2).

En parallèle, les étudiants de l'université de Masuku avaient transformé leurs salles de TP en musée et mis en place plusieurs expériences autour de la lumière, qu'ils ont pu présenter aux élèves des collèges et des écoles primaires avant les ateliers. 100 collégiens et 100 élèves d'écoles primaires ont pu assister à ces animations.

Entre ces ateliers, plusieurs conférences ont été proposées aux étudiants et personnels de l'université : "La mesure du temps : de la clepsydre à l'horloge atomique " par Marie Houssin (AMU), "À travers l'arc-en-ciel et au-delà " par Sandrine Ferri (AMU), "Physique du céleste " par O.T. Megadémini. Une centaine de personnes étaient présentes lors de chaque conférence, et les questions furent très pertinentes.

Cette semaine s'est close par une grande fête de la physique, le dimanche 3 novembre sur la place centrale de la ville. Les habitants s'en souviendront longtemps. Les étudiants de l'USTM menaient l'animation sur un podium en expliquant le phénomène de l'éclipse aux passants qui s'arrêtaient, intéressés par cette agitation inhabituelle (fig. 3). Les stands proposaient aux enfants des activités autour de la lumière, telles que la fabrication de cadran solaire, des disques de Newton en toupie, des explications sur le système solaire. De plus, des expériences optiques étaient présentées à l'écomusée voisin. 

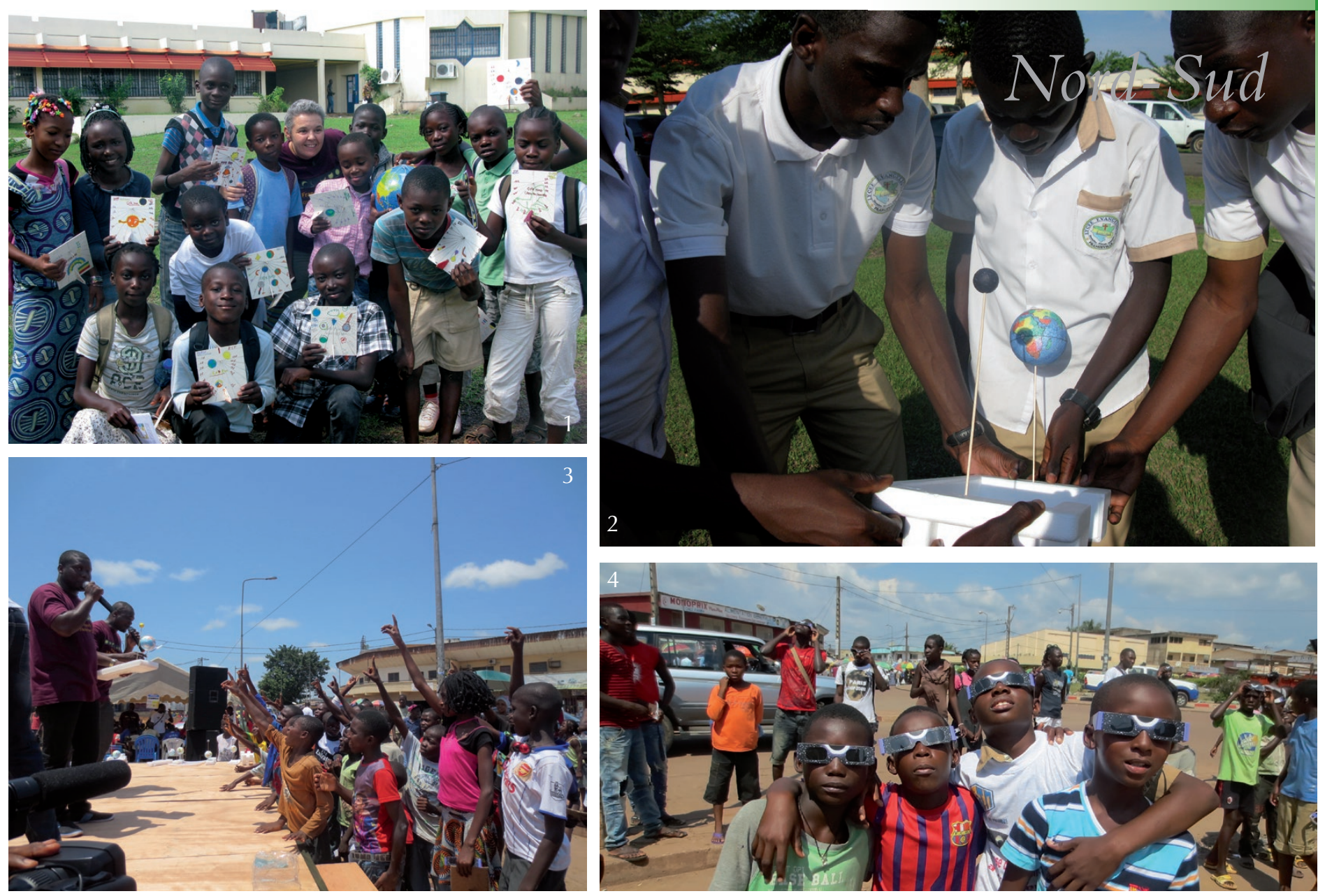

Par chance, la météo a été favorable pour observer le phénomène de l'éclipse, très peu de nuages sont venus voiler le ciel. Lorsque l'éclipse a commencé vers 13 h 30, les stands se sont arrêtés et les habitants ont tourné leur regard (bien protégé !) vers le soleil (fig. 4). La température et la luminosité ont diminué. Les ombres ont pris des formes très particulières. L'éclipse solaire vue de Franceville était maximale à 15 h 00 , avec une obscuration de 95,7\% (voir photos p. 34).

L'objectif de cette semaine était de démystifier la science en faisant entrer des écoliers dans l'université, en leur présentant de belles expériences d'optique, en leur faisant réaliser des cadrans solaires et en leur expliquant le phénomène de l'éclipse, objectif largement atteint ! Plus de 200 élèves ont participé aux ateliers. Les adultes ont aussi posé de nombreuses questions et la ville de Franceville, plutôt calme d'ordinaire, a connu une animation exceptionnelle. Plus de 2000 personnes se sont arrêtées devant le podium installé sur la place de la ville pour l'occasion et ont admiré la Lune "grignotant» le Soleil. Plus inattendu, l'effet le plus important était certainement celui produit sur les étudiants impliqués. Intérêt, motivation, plaisir d'enseigner, goût de l'interaction avec un public très vaste, transmission des savoirs, fierté d'avoir pu organiser un événement avec succès, les étudiants de tous les niveaux de l'USTM à Franceville sont les grands gagnants de cette éclipse.

Sandra Gely ${ }^{(1, *)}$, Marie Houssin ${ }^{(2)}$ (marie.houssin@univ-amu.fr), Martina Knoop ${ }^{(2)}$ et Alain Brice Moubissi ${ }^{(3)}$

(1) Institut d’Optique Graduate School, Palaiseau. (*) Adresse actuelle : société Optis, Lausanne, Suisse

(2) Laboratoire Physique des interactions ioniques et moléculaires, Université d'Aix-Marseille (3) Université des sciences et techniques de Masuku, Gabon

1. Écoliers de Franceville, fiers de leurs cadrans solaires magnifiquement décorés.

2. Observation de l'ombre de la « Lune » sur la maquette «Terre » réalisée par des collégiens de Franceville.

3. Le 3 novembre à Potos (quartier central de Franceville) : animations en attendant l'éclipse.

4. Jeunes Gabonais bien protégés observant l'éclipse.

\section{L'Université des sciences et techniques}

\section{de Masuku (USTM)}

L'Université des sciences et techniques de Masuku est un établissement public d'enseignement supérieur situé à Franceville dans la province du Haut-Ogooué, au sud-est du Gabon. Créée et organisée par la loi du 29 janvier 1986, elle comprenait initialement deux établissements pédagogiques, transférés de Libreville à Franceville : la Faculté des sciences et l'École nationale supérieure d'ingénieurs de Libreville (ENSIL).

L'USTM est aujourd'hui composée de plusieurs établissements :

- Faculté des sciences (chimie, biologie, géologie, mathématiquesphysique, physique-chimie)

- École polytechnique de Masuku (EPM)

- Institut national supérieur d'agronomie et de biotechnologies (INSAB)

Elle accueille 1520 étudiants, répartis dans sept licences et neuf spécialités de Masters recherche et professionnels. L'enseignement est en français.

L'USTM comprend six unités de recherche (chimie, agronomie et biologie, sciences de la Terre, mathématiques et informatique, physique, mécanique des matériaux), regroupant 22 laboratoires. L'USTM a un site internet tout neuf : http://univ-masuku.ga 